\title{
La querelle des noms. Chaînes et strates ethnonymiques dans le Chaco boréal
}

Nicolas Richard

\section{(2) OpenEdition}

1 Journals

Édition électronique

URL : https://journals.openedition.org/jsa/11935

DOI : 10.4000/jsa. 11935

ISSN : 1957-7842

Éditeur

Société des américanistes

Édition imprimée

Date de publication : 20 décembre 2011

Pagination : 201-230

ISSN : 0037-9174

\section{Référence électronique}

Nicolas Richard, «La querelle des noms. Chaînes et strates ethnonymiques dans le Chaco boréal », Journal de la Société des américanistes [En ligne], 97-2 | 2011, mis en ligne le 10 décembre 2014, consulté le 03 septembre 2022. URL : http://journals.openedition.org/jsa/11935 ; DOI : https:// doi.org/10.4000/jsa. 11935 


\title{
LA QUERELLE DES NOMS. CHAÎNES ET STRATES ETHNONYMIQUES DANS LE CHACO BORÉAL
}

\author{
Nicolas RICHARD *
}

Les noms ethniques, encore en vigueur en 1940, pour désigner les populations indiennes du Chaco boréal (Chulupí, Moro, Chamacoco, Lenguas...) ont été remplacés dans les décennies suivantes par une nouvelle strate de noms (Nivaclé, Ayoré, Ishir, Enlhet...) qui peuvent être traduits, dans tous les cas, par " les hommes» ou «les humains ». Ce bouleversement correspond-t-il uniquement à une transformation d'ordre nominatif ? Ou bien est-il corrélé à une évolution profonde dans l'agencement social et historique des populations indiennes du Chaco ? L'avènement de l'anthroponyme en tant que nom ethnique confirme-t-il la thèse du caractère ethnocentré de ces populations ou résulte-t-il, au contraire, du démantèlement des rapports interethniques, de l'occupation et de la colonisation définitive de ce territoire ? À partir des travaux réalisés par plusieurs auteurs sur les groupes du Chaco, cet article analyse les différentes strates ethnonymiques qui le composent, en partant de la plus récente pour remonter à celles de l'époque coloniale. Appelé la «Babel d'Amérique» par les jésuites du $\mathrm{XVIII}^{\mathrm{e}}$ siècle en raison de l'instable fragmentation linguistique qu'il présente, le Chaco deviendra, par là même, plus intelligible. [Mots-clés : Chaco boréal, Indiens du Chaco, ethnonymes, ethnohistoire, Paraguay.]

La disputa de los nombres. Cadenas y estratos etnonímicos en el Chaco boreal. Los nombres étnicos, todavía utilizados en 1940, para designar a los pueblos indígenas del Chaco boreal (chulupís, moros, chamacocos, lenguas...) fueron reemplazados progresivamente en las décadas siguientes por un nuevo estrato de nombres (nivaclés, ayoreos, ishirs, enlhets...) que pueden en todos los casos ser traducidos por «los hombres », " los humanos ». ¿ Se trata simplemente de una evolución nominal o de una transformación más profunda en la estructuración social e histórica de dichas poblaciones ? ¿ La elección del antropónimo como nombre étnico confirma la tesis del carácter etnocéntrico de estas sociedades o resulta, más bien, del desmantelamiento del sistema de relaciones interétnicas y de la ocupación y colonización definitiva del Chaco tras la guerra paraguayo-boliviana? A partir de trabajos realizados por distintos autores, este artículo analiza los diferentes estratos etnonímicos en el Chaco boreal

* CNRS, CERHIO-UMR 6258/Instituto de Investigaciones Arqueológicas y Museo, Universidad Católica del Norte, Chile, université de Haute-Bretagne, Rennes 2, place du recteur Henri Le Moal, CS24307,35043 Rennes cedex [nicolasrichardv@gmail.com].

Journal de la Société des Américanistes, 2011, 97-2, pp. 201-230. (C) Société des Américanistes. 
partiendo del más reciente y remontando a los de la época colonial. Llamado la « Babel de América » por los jesuitas del siglo XVIII en razón de su inestable fragmentación lingüística, el Chaco se volverá, a través de esta encuesta por ello mismo más inteligible. [Palabras claves: Chaco boreal, indígenas del Chaco, nombres étnicos, etnohistoria, Paraguay.]

The case against names. Ethnonymic chains and strata in the Northern Chaco. The ethnic names, still in use in 1940, to designate the indigenous peoples of the Chaco Boreal (Chulupi, Moro, Chamacoco, Lenguas...) were gradually replaced over the following decades by a new layer of names (Nivaclé, Ayoreo, Ishir, Enlhet...) all of which can be translated as "men », or « humans ». Is this simply a semantic evolution or a more profound transformation in the social and historical agency of these groups ? Does the advent of an anthroponym as an ethnic name confirm the ethnocentric character of these societies or is it more likely a result of the dismantling of inter-ethnic relations and ultimately of the occupation and colonization of the Chaco after the Paraguayan-Bolivian war? Based on work by different authors, this article analyzes the different ethnonymic strata in the northern Chaco beginning with the most recent ones and reaching back to those from the colonial era. As a result, the Chaco - which 18th century Jesuits called the "American Babel» because of its unstable linguistic fragmentation - will become more intelligible. [Key words : Chaco Boreal, indigenous people of the Chaco, ethnic names, ethnohistory, Paraguay.]

\section{LE BOULEVERSEMENT DES NOMS}

Un bouleversement profond du paysage ethnonymique du Chaco s'est opéré dans les dernières décennies ${ }^{1}$; un basculement général des dénominations ethniques qui ne semble pas avoir intéressé autrement les spécialistes, comme s'il était inscrit dans la nature des choses, comme s'il s'opérait dans le sens de l'histoire, sans poser de problème, ni offrir de résistance. En 1940 encore, Métraux pouvait décrire un Chaco peuplé de Chulupí, Moro, Chamacoco, Mataco et Chorote. Quarante ans plus tard, cette région est habitée respectivement par des Nivaclé, des Ayoré, des Ishir, des Wichí et des Manjui. Une coupure profonde sépare donc la " carte ethnique » utilisée par les militaires au cours de la guerre du Chaco (1932-1935) de celle qui est affichée de nos jours dans les musées ethnographiques ou dans les manuels scolaires, une coupure qui parcourt le Chaco de façon exhaustive et transversale, rendant mutuellement inintelligibles des nomenclatures et des index utilisés un siècle plus tôt. Ce mouvement a été assumé dans l'indifférence, comme s'il n'affectait en rien la nature de l'objet, comme s'il ne traduisait pas une transformation de fond dans la façon dont les identités indiennes du Chaco se définissent et s'agencent. Basculement massif donc, mais insignifiant, qui a fini par prendre la forme d'un non-événement. Il faudrait bien sûr comprendre les raisons de cette banalisation. Accordons-nous d'abord sur la nature des faits. 
Commençons par comparer deux cartes ethnographiques, une de celles qui pouvaient encore être en usage vers 1950 ( « strate moderne ») et une autre utilisée de nos jours pour représenter la diversité ethnique du Chaco boréal («strate contemporaine ») (Figure 1). La carte de Bélaieff (1941) et celle de Métraux (1946a) sont exemplaires pour la première strate ; une autre, utilisée actuellement à des fins pédagogiques dans les musées et institutions indigénistes paraguayennes, serait paradigmatique de la seconde (Zanardini et Biederman 2001). Simplifionsles, même au risque de perdre quelques détails géographiques, afin de mieux cerner leurs articulations principales.

Sur les rives du Pilcomayo, en s'éloignant d'Asunción, les groupes qui étaient encore récemment connus sous le nom de Toba sont à présent appelés Qom, ethnonyme utilisé pour désigner aussi bien les anciens Toba occidentaux qui habitaient le haut Pilcomayo que les Toba orientaux plus proches de son embouchure dans le Paraguay. Les groupes qom vivant sur la rive paraguayenne du Pilcomayo précisent leur identité par un «Qom-lik» (Zanardini et Biederman 2001). Sur le Pilcomayo encore, en amont des Esteros de Patiño, les groupes qui étaient connus comme « Ashluslay » (Nordenskiöld 1912), " Sociagay » (Belaieff 1941) ou "Suhín » (Grubb 1911) et, plus généralement, comme "Chulupí » (Métraux 1946a) sont à présent appelés « Nivaclé », terme rapporté pour la première fois par Súsnik en 1961. De même, en amont du pays nivaclé, approchant la frontière bolivienne, les groupes qui étaient encore récemment connus comme Chorote se réclament à présent du nom de «Manjui » (Zanardini et Biederman 2001), mais aussi, très récemment, de celui de "Lumnanas» (Chamorro 2006). En s'avançant vers le nord, en remontant l'actuelle frontière bolivienne, des groupes détachés de ceux que Nino (1912) appelait encore « Chiriguano » et qui furent appelés Guarayo lors de la guerre du Chaco, puis Guarani occidentaux dans les décennies qui suivirent, préfèrent à présent se nommer simplement Ava ${ }^{2}$. Ceux que Métraux (1946b) appelait « Tapieté » (et qui continuent, côté argentin, à se nommer ainsi) sont devenus, au Paraguay, des Guarani Ñandeva, mais préfèrent, eux aussi, la désignation générique de Ava ou Guarani. En revenant vers l'est, au centre du Chaco, au sud des anciennes missions jésuites de Chiquitos, la constellation des populations jadis connues sous les noms de Tsirakua, Ugaraño, Guarañoca et, plus généralement, à partir de la guerre du Chaco, Moro, sont à présent unanimement connus sous le nom d'Ayoré ou Ayoreo, terme qui fut pour la première fois identifié par Haeckel (1955), puis repris par Kelm (1960) et Sebag (1964; 1965). Appartenant à la même famille linguistique, installés sur la rive occidentale du Haut Paraguay face au Pantanal brésilien, les Chamacoco, dont le nom fut consigné pour la première fois à la fin du $\mathrm{XVIII}^{\mathrm{e}}$ siècle (Rodrigues do Prado 1795) et repris plus tard par Boggiani (1894), Baldus (1927), Súsnik (1957) ou Bernand (1977), retiennent à présent la dénomination d'Ishir, terme qui fut progressivement introduit dans la littérature ethnographique depuis Cordeu (1974) et récemment assumé, par 


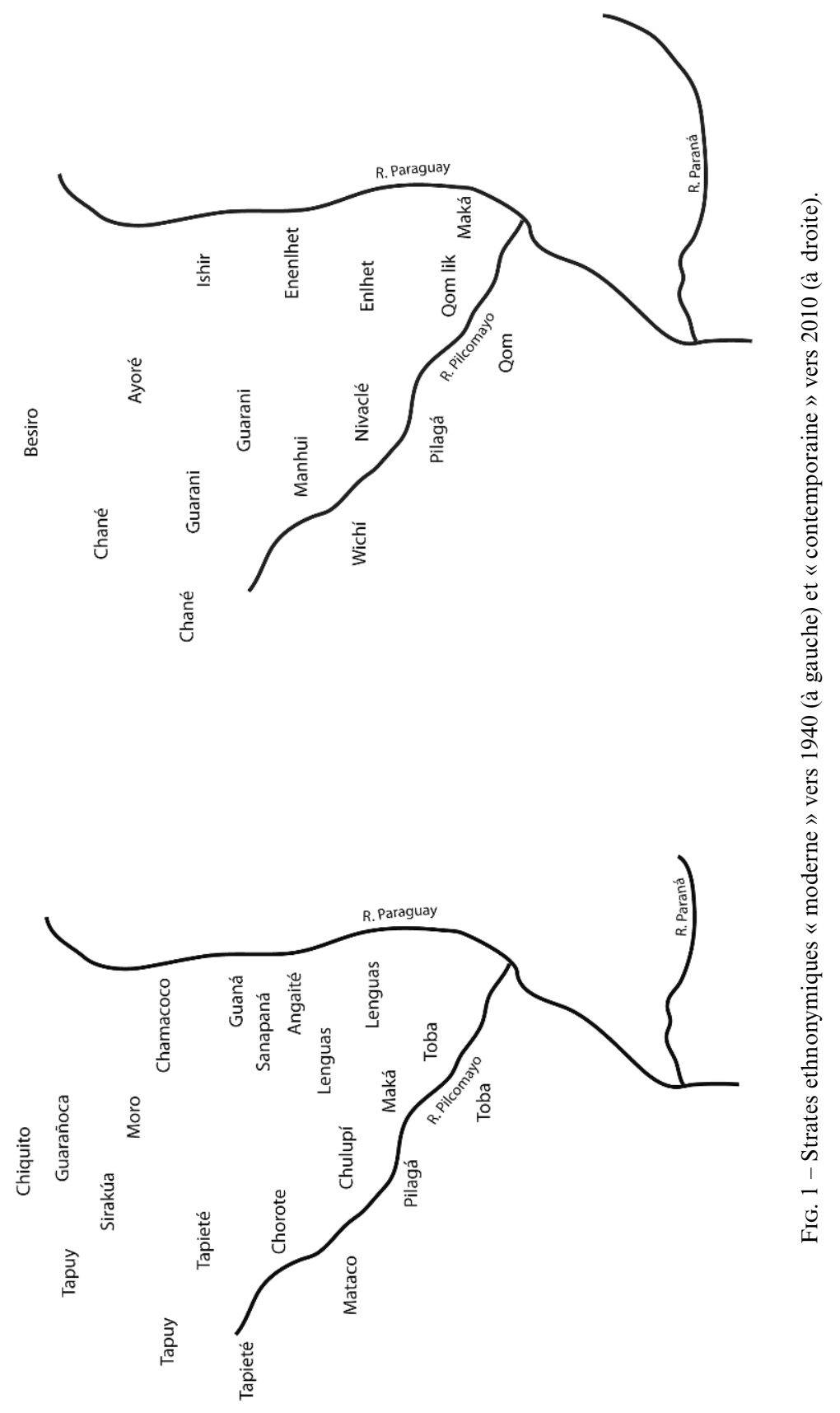


exemple, par Escobar (1999). En outre, plusieurs groupes jadis connus comme Lengua, Guentuse, Guaná, Sanapaná, Sapuki ou Angaité (entre autres) sont aujourd'hui regroupés sous les noms d'Enlhet et d'Enenlhet au nord du département de Presidente Hayes et au sud de celui d'Alto Paraguay (Unruh et Kalish 2003 ; Fabre 2005-2007).

C'est donc massivement que les dénominations indigènes basculent, au cours des dernières décennies, vers une nouvelle ethnonymie. On voit bien l'ampleur de ce basculement: presque aucun groupe indien du Chaco n'y a échappé. Ce glissement s'est d'abord produit dans la littérature spécialisée (approximativement, entre 1960 et 1980) et a été plus tard repris et confirmé par la législation paraguayenne et les acteurs institutionnels, notamment à partir des années 1990 . Cependant, malgré son ampleur et son caractère immédiatement visible, ce mouvement n'a guère attiré l'intérêt des ethnologues et des spécialistes de la région. Au contraire, ce passage a été assumé par les différents acteurs du champ ethnique comme s'il s'agissait d'une évolution normale de la situation, un ajustement ou un recadrage des anciennes taxonomies devenues désuètes, une sorte de clarification salutaire du panorama ethnologique du Chaco. Trois types de raisons nous semblent pouvoir expliquer cette absence de problématisation.

Premièrement, cette évolution a été généralement interprétée en terme de morale. Il s'agirait, dans tous les cas, d'un passage allant du « faux » au « vrai ». En effet, nous le verrons, le nom retenu se voudrait être un « autonyme » ou un « endonyme » (nom que le groupe se donne à lui même), tandis que celui qui est déchu serait un " exonyme ", en ceci qu'il fut attribué par un tiers, indien ou pas, puis réifié ou fixé par le front de colonisation, les militaires ou les appareils d'État. Ainsi, le sujet indigène, toujours collectif et toujours égal à lui-même, aurait traversé la jungle touffue des dénominations erronées et des noms aliénants pour conquérir, en fin d'histoire, le droit d'énoncer lui-même, dans la transparence retrouvée de son identité, son propre nom, son nom propre. L'histoire des noms indiens serait donc l'histoire d'une aliénation et d'un malentendu dont ils seraient (et nous serions) enfin en train de sortir. Ce mouvement irait donc dans le sens d'une émancipation. Nous ne nous prononcerons pas sur la pertinence politique ou éthique de cet argument, mais il nous intéresse d'en montrer ses limites heuristiques. Elles tiennent, d'une part, à ce que des noms ne sauraient être vrais ou faux : les structures de nomination qui articulent le Chaco au XVIII ${ }^{\mathrm{e}}$ siècle ne sont pas plus fausses, ni plus vraies, que celles qui ont cours de nos jours. Ces structures de nomination fonctionnent et, puisque la plus grande partie des sources historiographiques disponibles pour travailler sur la région consiste en des noms rapportés par des chroniqueurs, des missionnaires ou des militaires au gré de leurs explorations, il conviendrait, non pas de les juger par rapport à leur degré de véracité, mais d'expliquer comment ils ont pu être institués, dans le cadre de quelle relation et pour traduire quels rapports. Ces matériaux ne sont exploitables qu'à la condition de désamorcer cette opération par laquelle toute 
considération sociologique est évacuée au profit d'une énonciation morale et humaniste du problème. Entendons-nous bien sur ce point : il ne s'agit aucunement pour nous de contester les termes actuellement utilisés par les communautés indiennes pour se nommer, puisque tout collectif a par définition le droit de décider de son nom. Par contre, cette décision ne saurait se substituer à l'analyse raisonnée des conditions sociales et historiques qui l'ont permise ou incitée.

Deuxièmement, ce basculement ethnonymique est devenu invisible parce que sa portée et son seuil d'émergence n'apparaissent qu'à la condition de regarder cette région dans son ensemble et, donc, de sortir du cadre monographique - un ethnologue, un texte, un groupe. À aucun moment, ce passage n'a été pensé comme un mouvement transversal touchant simultanément l'ensemble des groupes indiens du Chaco. Au contraire, l'on trouvera un même argument s'exerçant séparément pour chacun des groupes en question, sans que le caractère général du mouvement ne soit jamais énoncé, comme si son niveau d'émergence était l'histoire particulière de tel ou tel groupe. Ainsi, nous trouvons, là, un examen détaillé des "faux noms » prêtés aux « Nivaclé » qui conclut à la nécessité de garder ce dernier terme (Chase-Sardi 2003), plus loin, une argumentation en règle pour le bannissement d'un «Chamacoco » qui nous a trop longtemps trompé (Sequera 2005), ailleurs, une longue exégèse sur la nécessité de passer du «Sanapaná » à l'actuelle dénomination d' " Enxet » (Unruh et Kalish 2003). Autrement dit, ce mouvement a été pensé dans le cadre des approches monographiques produites par l'ethnologie régionale dans la dernière moitié $\mathrm{du} \mathrm{xx}^{\mathrm{e}}$ siècle. À vrai dire, non seulement ce mouvement est pensé dans un cadre monographique, mais encore, l'un et l'autre constituent en quelque sorte deux dimensions d'un même mouvement. N'est-ce pas dans la première monographie qui leur fut dédiée (Susnik 1961) que le terme « Nivaclé » fait surface ? N'est-ce pas avec la production du premier corpus monographique (Haekel 1955 ; Kelm 1960 ; Sebag 1964, 1965 ; etc.) sur les Ayoré que ce terme est apparu? Une mise en scène bien particulière voudrait que l'ethnologue, perçant pour la première fois le « monde intérieur » du groupe en question, découvre - ce que nul n'avait pu faire avant lui - le véritable nom de ceux qu'il est en train d'étudier et non plus celui, extérieur, que lui prêtait l'ignorance des colons, des missionnaires, des militaires ou des explorateurs qui l'ont précédé. Dans ces conditions, la question du caractère général et transversal de ce mouvement n'a pas pu être posée et, de la sorte, ses ressorts historiques, institutionnels et sociologiques n'ont pas pu être calibrés au niveau qui est le leur : non pas celui de tel groupe en particulier, mais celui de la façon dont s'organisent et s'agencent les différences ethniques en un moment historique déterminé.

Enfin, troisièmement, cette invisibilité résulte aussi d'une sorte de discrédit à la base du phénomène même qu'il s'agirait d'expliquer. Ainsi, nous dira-t-on, il n'y a pas, à proprement parler, de changement de nom. Il s'agirait plutôt d'une " découverte », du « dévoilement » d'une vérité jusqu'ici cachée, enfouie sous des 
formes aberrantes et extérieurement prêtées. Le nom a toujours été là, il n'a pas changé, seulement nous ne pouvions ou ne voulions pas le voir. Nous nous occupons donc d'un faux problème, puisque nous serions en train de confondre deux ordres de réalité différents : celui des faits ethnologiques, d'une part, qui se seraient maintenus stables - ce nom qui était toujours déjà là - et celui de notre appréciation de cette réalité, d'autre part, qui aurait en revanche évolué - nous sommes enfin capables de le saisir ou de l'identifier. Il n'y a donc pas à proprement parler de " problème » ethnologique, mais une querelle nominale, ne concernant en rien la réalité de ce qui est nommé, un problème d'observateurs qui est extérieur à la nature de l'objet. Mais, est-ce vraiment le cas ? Nordenskiöld aurait séjourné une année durant parmi les Ashluslay sans que personne ne lui dise qu'il était en fait chez les Nivaclé ? Les missionnaires anglicans auraient évangélisé pendant des années des Lenguas sans se rendre compte qu'ils étaient en train de prêcher à des Enlhet? Métraux aurait sauvé la vie de quelques Chulupí sans qu'ils aient eu la générosité de lui transmettre leur vrai nom ? Et Boggiani aurait pris une femme chamacoco et se serait fait assassiner par ses beaux-frères chamacoco sans que personne dans la famille ne daigne lui dire qu'ils n'étaient pas des Chamacoco ? Tous ces gens-là auraient donc attendu la venue des ethnologues contemporains pour leur révéler une vérité jusqu'alors soigneusement cachée et dissimulée au regard des visiteurs inexperts ? Trop enclins à effacer le rendement historique de leurs propres machines théoriques et institutionnelles, les acteurs du champ indigéniste ont promu ce passage au « vrai nom » à force d'imaginer une double transparence : la leur, d'abord, puisqu'ils ne seraient là que pour s'effacer (vieux rêve d'avant-garde) et nous livrer une compréhension immédiate et transparente de l'autre; celle des sociétés indiennes, ensuite, unanimes dans leur définition, égales à elles-mêmes dans le temps, privées de tout principe de contradiction et de toute politique du nom. Nous soutiendrons tout au long de ces pages une hypothèse différente, à savoir que l'évolution des noms et celle des réalités sociologiques qu'ils désignent sont mutuellement imbriquées de façon à ce que les transformations du tissu ethnonymique traduisent des évolutions profondes dans l'agencement social et historique des populations indiennes du Chaco.

\section{QUE DES « HOMMES »}

Nous avons déjà constaté que ce passage du « faux » au « vrai » nom s'opère de façon à peu près simultanée dans l'ensemble de la région. Cette simultanéité épuise-t-elle l'identité d'ensemble de ce mouvement? Il semble que non. Pour le montrer, il faudrait pouvoir contourner cette disposition courante : en effet, en règle générale, bien que la littérature spécialisée se soit largement dédiée, dans les dernières décennies, à rendre possible une traduction poussée des univers linguistiques et symboliques indiens, en produisant par exemple des compilations 
bilingues de récits, des dictionnaires ou des traductions en langue indienne de matériaux pédagogiques (mais aussi et surtout évangéliques), les noms indiens ont été épargnés. Comme si, en fait, pour qu'il puisse fonctionner en tant que nom, le terme retenu devait ne pas être traduit. Ou encore, en effaçant la signification du terme pour ne garder que sa trace linguistique, c'est la langue en elle-même qui est en quelque sorte réifiée et qui devient le critère fondamental de différenciation. Essayons donc à présent d'inverser cette opération et restituons, lorsque cela nous est possible, la signification vernaculaire du terme retenu : nous perdrons en ésotérisme ce que nous gagnerons en clarté. L'exercice ne se veut point exhaustif, il devrait seulement nous permettre de dégager un mouvement de fond.

Prenons quelques exemples, à commencer par ces mêmes Nivaclé que nous avons déjà mentionnés. Ces populations avaient leurs campements sur la rive gauche du Pilcomayo, en amont des Esteros de Patiño, et leur territoire pénétrait sur une centaine de kilomètres vers l'intérieur du Chaco, au sud et à l'ouest des actuelles colonies mennonites (Sterpin 1993; Chase-Sardi 2003; Siffredi et Santini 1993). Les premiers travaux significatifs qui leur ont été dédiés sont ceux du suédois Nordenskiöld $(1912 ; 1929)$ qui visita à plusieurs reprises leurs campements au début $\mathrm{du} \mathrm{xx}^{\mathrm{e}}$ siècle. Il ne s'agissait pas alors de Nivaclé, mais d'Ashluslay, terme que l'explorateur suédois fera entrer dans les annales et les index de l'ethnologie moderne. Ces Ashluslay seront aussi, et plus généralement, identifiés comme des Chulupí, nom sous lequel ces populations étaient connues sur le front de colonisation argentin. D'autres noms leur furent aussi prêtés, comme « Sociagay » (Belaieff 1941) ou " Suhin » (Grubb 1911), jusqu'à ce qu'en 1961 Susnik introduise le terme "Niwaquli», qui sera ensuite repris par les différents ethnologues qui se sont essayés sur ce dossier. Súsnik signale que ce terme voudrait dire « les hommes », définition sur laquelle revient Chase-Sardi (2003, pp. 98-99) : «Ils s'appellent eux mêmes Niwaklé [...] Niwaklé signifie "homme", avec un sens générique, être humain ".

Dans des circonstances différentes, une même évolution est à la base de l'apparition du terme « Ayoré ». Dans la frange septentrionale du Chaco, une constellation de groupes extrêmement réduits qui nomadisaient dans le vaste désert qui s'étend au sud des anciennes missions jésuites de Chiquitos furent connus dès le $\mathrm{XIX}^{\mathrm{e}}$ siècle sous des termes hétérogènes comme "Guarañoca ", «Tsirakúa » ou « Empelotas ». Au xx ${ }^{\mathrm{e}}$ siècle, ces populations furent plus généralement connues à travers le terme chamacoco "Murro» (hispanisé en « Moro »), répandu par l'armée paraguayenne lors de la guerre du Chaco. Le terme « Ayoré » («Ayorei », « Ayoreo ») est pour la première fois relevé dans la Pedagogical grammar écrite par les missionnaires protestants en 1955 (New Tribes Mission 1955). Il a été repris, depuis lors, par l'ensemble des travaux sur ces groupes. Ainsi, nous savons que « les Ayorés se nomment eux mêmes "Ayoreode", qui est la forme plurielle de ayorei, "l'homme", dont le féminin est "ayoré". 
Le terme "ayorei" s'emploie aussi pour désigner de façon générique tout être à figure humaine ${ }^{3}$ (Bormida et Califano 2003, p. 19).

À l'est du Chaco, à partir de la rive droite du fleuve Paraguay jusqu'aux abords des actuelles colonies mennonites dans l'intérieur du pays, des groupes jadis connus sous le nom de Mascoy (Mashcoi, Macikoí), qui incluaient des entités socio-ethniques aux dialectes rapprochés comme les Sanapaná, les Angaité, les Kaskiha, etc. (Métraux 1946b), ont été, depuis peu, réunis sous les désignations de Enlhet et de Enenlhet. Selon Unruh et Kalish (2003, p. 208), ces termes « résultent du fait que tous ces groupes partagent une notion semblable pour exprimer le concept de "personne humaine, homme" ", de sorte qu'elle est aujourd'hui utilisée comme autodénomination : "Dans ses propres termes, donc, la nation Enlhet-Enenlhet est "la nation des gens" ".

Nous pourrions multiplier les exemples : « Ishir », d'utilisation récente parmi les anciens Chamacoco, voudrait, lui aussi, dire «personne » ou « humain » (Cordeu 1981, p. 23), tout comme le « Qom » des anciens Toba ou le « Wichí » de ceux qui étaient auparavant identifiés comme Mataco ; le même constat s'impose pour le terme "Ava » utilisé par les Guarani occidentaux ou les Guarani ñandeva, termes qui sur-écrivaient déjà un ancien "Chiriguano » désuet. Ainsi, si on traduit les termes indiens qui organisent actuellement le paysage ethnonymique du Chaco, le résultat est bien paradoxal: tout le monde ou presque a pour nom ethnique, c'est-à-dire pour trait de distinction, quelque chose qui ressemble à « les gens », « les hommes » ou « les humains », forme commune et inclusive à tous.

La carte résultante aurait ceci de paradoxal qu'elle pourrait représenter n'importe quel paysage humain dès le moment où l'on convient que ces espaces sont fondamentalement habités par... « des gens ». Sauf si on donne à ces termes une dimension excluante. Et, en effet, pour les tenants du caractère ethnocentrique des populations concernées, le fait que ces populations se nomment elles-mêmes «les humains» ou «les personnes" supposerait inversement qu'elles nieraient cette qualité aux groupes environnants : si les Nivaclé se disent eux-mêmes "les humains », c'est bien parce que leurs voisins ne le sont pas, dit-on, ou alors ils le sont dans une moindre mesure.

De bien graves conclusions ethnologiques ont ainsi été tirées de cette disposition. Comme ce phénomène est attesté pour l'ensemble des populations de la région, nous voilà invités à nous rendre à la force de cette évidence et à conclure que chacun de ces groupes aurait vécu, depuis toujours, en une sorte de déni de l'humanité des autres. Cette idée est venue en renforcer une autre, selon laquelle la conception du monde de ces populations serait profondément, structurellement, ontologiquement ancrée dans leur particularité linguistique. Au-delà de leurs sociétés, c'est-à-dire au-delà de leurs langues, s'étendrait un espace inintelligible et opaque, peuplé d'êtres qui "ne sauraient pas parler» et qui appartiendraient, en conséquence, à des catégories sous-humaines ou pré-humaines. 
L'argument ethnocentrique se greffe alors sur un autre, qui ferait de la langue et de la communauté linguistique le socle de toute identité. Ce ne serait donc qu'à travers une éthique universelle - que telle ou telle civilisation aurait su imposer que les gens en seraient venus à se convaincre de l'humanité des autres. Et, comme cette éthique ferait défaut, en dehors des frontières du « soi » il ne pourrait y avoir que la guerre. Ce triple argument - déni d'humanité de l'autre, ancrage linguistique du soi, omniprésence de la guerre - fut très tôt théorisé : l'avancée évangélique sur le Chaco s'est justifiée sur les propositions inverses (propagation d'une éthique universelle, intelligibilité mutuelle des langues par référence à un seul Texte, reconnaissance de l'humanité de l'autre), et il est important d'insister sur la continuité, pas toujours affichée, qui existe entre la théorisation missionnaire de la question indienne et quelques-uns des maîtres arguments de l'ethnologie qui a été pratiquée dans la région. Ainsi donc, le « vrai nom » que ces communautés se donnent elles-mêmes est venu renforcer cette idée selon laquelle ces sociétés auraient vécu et vivraient encore dans une sorte d'autarcie métaphysique, incapables de toute compréhension de l'autre (privilège de la "science », dirait Lévi-Strauss), étanches à l'extérieur parce que transparentes vers l'intérieur. Voici donc le Chaco qui en résulte : une dizaine d'humanités parallèles, éparses dans l'espace, évoluant dans l'intimité ontologique de leur propre « cosmovision », attrapées dans une existence culturelle linguistiquement circonscrite, se faisant la guerre lorsqu'elles se rencontrent, s'ignorant lorsqu'elles ne le font pas, croyant être seules dans le monde parce qu'incapables de reconnaître dans l'autre un égal.

Sauf que rien, en dehors des nécessités de l'argument, ne permet d'affirmer le caractère excluant des anthroponymes - « les humains », " les hommes »-dont il est question. Bien au contraire, dès qu'on s'arrête sur le détail, des circulations sociales et culturelles, des dynamiques politiques et économiques, des zones mixtes et multivalentes tissent cet espace comme un continuum social qui ne se laisse point modeler par les frontières que lui trace la raison ethnographique. Les grands foyers de population qui ont structuré le Haut Paraguay n'étaient-ils pas composés de populations extrêmement hétérogènes - arawak, zamuco, otuqui bororó, guaicurú - intégrées en un même système économique, social et politique ? L'énorme quantité d'intermariages nivaclé-enlhet ou nivaclé-maká, attestés déjà par les premiers observateurs, doivent-ils être compris comme une déviation, une exception ou une dérogation à la règle ? Dans le Chaco, le multilinguisme est une condition normale - initiale -, les mariages interethniques sont extrêmement fréquents, la circulation des individus entre les différents groupes est, elle aussi, normale; des relations économiques et politiques à caractère multiethnique sont la règle, des nucléations regroupant des individus d'origine hétérogène sont fréquentes et nul ne semble hésiter autrement si, avançant par un chemin de traverse, il venait à butter contre les limites de son " humanité ». Il apparaît très vite que la densité et l'intensité des rapports inter-linguistiques et inter-groupaux dans le Chaco rendent pour le moins 
problématique cette proposition selon laquelle les termes comme « les hommes » ou « les humains » traduiraient un « déni d'humanité de l'autre » ou un ethnocentrisme farouche et caractéristique.

Au contraire, on peut poser l'hypothèse que l'utilisation de l'anthroponyme comme nom ethnique signale, non plus cet excès de différence qui consisterait à s'adjuger l'exclusivité du genre humain, mais, au contraire, un défaut, une dilution ou une neutralisation de ces différences. Car il apparaît que, par un mouvement qui est aussi général à l'ensemble de la région, ces termes ont fini par signifier, non pas seulement «les hommes» ou «les personnes", mais plus précisément «les Indiens» ou « les Indigènes", par opposition aux «Paraguayens » et aux "non-Indiens » en général. Il en est ainsi du terme « Ishir ». Si, comme nous l'avons déjà signalé, ce terme veut dire littéralement « les personnes » ou « les humains », il est aujourd'hui fréquemment utilisé pour désigner par extension « les Indiens ». Le pluriel « Ishiro » s'oppose alors très nettement aux « Paraguayens », principal alter du cadre politique local. Ainsi, lorsqu'un Ishir cherchera à se démarquer d'une autre population indienne, il utilisera " Ishir kenaho », c'est-à-dire littéralement " autres Indiens » (Richard 2008a). Blaser (2004), étudiant la configuration du pouvoir local dans la région, tire des conclusions semblables. De même, le dictionnaire ishiro-español, publié par la mission évangélique de Puerto Diana (Ulrich et Ulrich 2000) inclut, à côté de la traduction habituelle de ishir comme " homme ", cette autre acception: « indigène ». Chase-Sardi (2003) note que le terme " nivaclé » est, lui aussi, fréquemment utilisé pour désigner extensivement « les Indiens » dans le cadre des relations avec les colonies mennonites ou autres exploitations agricoles. Le glissement est encore plus notoire dans le cas du terme « Ava ». Dans le Paraguay oriental, ce terme en vint très tôt à signifier, non plus « les hommes » comme le voudrait son étymologie, mais bien « les Indiens » en sens générique ${ }^{4}$. Dans le Chaco, les Guarani occidentaux (anciennement Guarayo ou Chiriguano) et les Guarani ñandeva (anciennement Tapieté) préfèrent aujourd'hui s'identifier par ce terme "Ava » qui, au risque d'effacer la distinction nominale entre les deux groupes, prend aussi le sens générique d' "Indiens » ou " indigènes », gardant comme seul sens distinctif celui qui oppose ce terme aux « non-Indiens ». Enfin, c'est dans ce sens aussi que vont Unhru et Kalish (2003) lorsqu'ils signalent que le terme « Enlhet », qu'ils traduisent par « personnes » ou par « gens », doit aussi être traduit par « indigènes"

Ainsi la strate émergente des noms ethniques traduirait un dépassement des anciens clivages groupaux. Car, à première vue, s'il fallait se rendre à une évidence, il apparaît que des termes comme « les personnes » ou " les hommes » constituent les catégories les plus inclusives possibles. Elles ont une fonction qui se situe à l'opposé de celle qu'un «nom» devrait avoir. Elles confondent cela même qu'un nom serait appelé à différencier. Est-ce vraiment un nom, « des personnes »? En quoi consisterait ce «nom » qui dilue toute spécificité du soi 
dans une condition générale et commune à tous ? Ce mouvement n'est pas univoque, il installe une ambiguïté, un « jeu » dans l'utilisation et la signification du terme. En tout cas, une tendance d'ensemble se dégage, qui montre comment ces termes perdent progressivement leur capacité distinctive à l'intérieur du monde indien pour fonctionner comme marqueurs identitaires recouvrant la fracture qui s'installe entre ce monde et les acteurs non indiens. C'est-à-dire qu'ils ne répondent plus aux articulations politiques et interethniques qui organisaient de longue date le Chaco indien, mais qu'ils traduisent une nouvelle configuration et un nouvel agencement du champ. Si «Chamacoco » pouvait jouer de sa différence avec «Moro», c'est qu'une ancienne fracture faite d'hostilités mutuelles séparait jadis les deux camps : l'extension inclusive d'Ishir (utilisé comme « Indien ») résulte, d'une part, de l'obsolescence effective de ce clivage, de l'autre, de l'avènement d'un nouveau champ politique et relationnel clivé sur la distinction entre «Indien » et « non-Indien ».

Il y a donc deux façons d'interpréter cette carte - « les gens », " les gens », etc. L'une procède en forçant ethnographiquement le caractère excluant de ces termes pour brandir la thèse de l'ethnocentrisme indien ; l'autre, prenant « ces gens » à la lettre, y verrait, au contraire, un affaiblissement et un dépassement des anciens clivages interethniques.

\section{« LES INDIENS »}

Mais alors, qu'en est-il de la strate ethnonymique déchue ? Quels sont ces autres noms que le « vrai nom » est venu effacer? Quelles sont les relations et les médiations que traduisait l'ancienne strate et dont la nouvelle signalerait l'extinction? Comme ci-dessus, commençons par essayer, lorsque cela est possible, de traduire ces termes et de leur restituer un sens. Une fois de plus, l'exercice ne se veut pas exhaustif : nous cherchons seulement à dégager la forme générale du problème.

Revenons à ce « Nivaclé », " les hommes », retenu par Súsnik en 1961. Ce terme, on l'a vu, fut précédé par une série de noms dont nous pouvons tracer un inventaire préliminaire. Si l'on écarte les ethnonymes utilisés dans les sources du $\mathrm{XVIII}^{\mathrm{e}}$ siècle pour ne retenir que ceux qui étaient en usage au moment de la guerre du Chaco, le résultat est déjà suffisamment impressionnant. Ainsi, Métraux (1946b, p. 235) recense l'utilisation des termes «Chunupí », «Chulupí », " Chorote », « Sówa », « Sówuash », « Suhin », " Sotiagai », « Sotegaraik », «Etehua » et « Tapieté ». Nordenskiöld, qui leur dédie un ouvrage en 1912, les nomme "Ashluslay». Chase-Sardi (2003), reprenant Métraux, ajoute les termes «Churupí », "Chunipí » et «Chotiagai », et Susnik (1978) celui de «Lateshelechí ». Les missionnaires Grubb (1911) et Hunt (1915) utilisent le «Suhin » recensé par Métraux. Nous ne pouvons fournir une traduction pour 
l'ensemble de ces termes, mais le sens de quelques-uns nous est connu. Ainsi, nous savons que « Ashluslay » (déformation de « Alhu'lhai ») serait un terme chorote (mais les Chorote sont à présent des Manhui) désignant les campements nivaclé les plus occidentaux et voulant dire quelque chose comme " gueules d'iguane » (Chase-Sardi 2003, tome 1, p. 113 ; Bossert et Siffredi 2011, p. 41). " Lateshelechí » serait, quant à lui, un terme mataco (mais les Mataco sont à présent des Wichí) dérivé de « latsich » (maiis) et signifiant quelque chose comme « avec du maïs » (Susnik 1978, p. 123). De même, « Chulupí » serait la déformation hispanisante d'un mot wichí, sona-pe ou chona-pe, voulant dire « avec des moutons » (Susnik 1981, p. 189 ; Chase-Sardi 2003, tome 1, p. 98). « Sociagay » est la déformation du terme toba «Sotirgaik » (mais les Toba sont aujourd'hui des Qom) : il reprend probablement le terme pilagá « Chotiagaï », mais sa signification précise nous échappe. "Guentuse », «Gentuse » seraient, pour leur part, des termes maká, avec ceci d'intéressant qu'ils auraient été utilisés aussi bien pour désigner les campements maká les plus occidentaux que les campements nivaclé les plus orientaux, tous deux confondus sous une même désignation (Chase-Sardi 2003, p. 98). Rappelons que Wichí (Mataco), Manhui (Chorote), Nivaclé et Maká participent d'un même continuum linguistique et social (famille linguistique mataco-mataguayo).

La traduction des termes utilisés sur la marche occidentale du Chaco s'avère être un peu plus aisée du fait que ce sont en général des termes guarani (chiriguano). Ainsi, le sens de «Tapieté » nous est bien connu : en guarani, cela voudrait dire quelque chose comme « vrais esclaves », " très esclaves » ou " plus esclaves »(Combès 2008), nuance qui le distingue du «Tapui » utilisé pour nommer les populations chané et qui signifierait, à son tour, " esclave » ou «serf» (Combès 2005). Ce même terme chiriguano - «tapuilesclave » - se décline encore en une troisième dénomination tapuy miri, « petit esclave », qui fut probablement à l'origine du nom de «Chiquitos» (" petits», en espagnol), traduit du chiriguano par les jésuites, et utilisé pour nommer un ensemble hétérogène de populations vivant dans l'actuelle Chiquitanía à l'est-nord-est de la ville bolivienne de Santa Cruz de la Sierra. Au nord des « Chorote », donc, nous trouverions successivement des « vrais esclaves », des « esclaves » tout court et enfin des « petits esclaves ». L'ensemble de ces termes est employé dans le guarani des Chiriguano. Le mot « chiriguano » a, quant à lui, fait l'objet de plusieurs hypothèses. La plus couramment retenue, par exemple par Pifarré (1989), fait de chiriguano une expression quechua voulant dire quelque chose comme " merde froide », « excrément froid » ou, moins crûment, " châtiés par le froid ». Cette étymologie est contestable, car toute une série d'éléments la rend historiquement problématique. En premier lieu, le fait que les sources anciennes notent systématiquement « chiriguaná » (ou le pluriel hispanisé « chiriguanaes ») et non pas " chiriguano ». Le glissement entre ces deux graphies se serait opéré au cours du $\mathrm{XVIII}^{\mathrm{e}}$ siècle et aurait permis (ou aurait été permis par) le développement de la 
folk-etymology «merdes froides ». En ce sens, en se référant au terme ancien, Combès et Saignes (1991) proposent d'en faire la contraction entre « chiriones » (terme guarani désignant des « métis ») et « guanás » (nom donné aux populations chané-arawak du Haut Paraguay) : chiri-guaná voudrait donc dire quelque chose de proche de « métissés avec des Guaná » ${ }^{5}$. Nous ne pouvons évidemment pas trancher dans cette affaire, mais il nous intéresse de constater que, dans tous les cas, (1) «Chiriguano » n'est pas un terme de la langue chiriguano (guarani) et (2) le terme a un caractère péjoratif.

Plus vers l'intérieur du Chaco, les actuels Ayoré étaient connus, sur la frange occidentale, par des termes comme « Tsirakúa », " Yanaigua », « Guarañoca » ou «Empelotas». Le terme «Tsirakuá », consigné pour la première fois comme un nom d'« ethnie » par Nordenskiöld (1912) qui rencontra une vieille captive tsirakuá dans un campement tapieté, semble n'être autre que le siracua chiriguano, qui signifie « piteux » (Romano et Cattunar 1916, p. 193). «Yanaigua » nous est mieux connu, il équivaudrait au «Kaaingua » des Guarani du Paraguay oriental, voulant dire, dans les deux cas, «ceux de la brousse ", " sauvages » ou, encore, « sauvages de la brousse », etc. (Combès 2004). C'est aussi dans ce sens qu'est employé le « Empelotas » répertorié dès la fin du XIX ${ }^{\mathrm{e}}$ siècle et utilisé, entre autre, par Métraux (1946b). Le métaplasme « Empelotas » cache mal l'espagnol en pelotas, littéralement « à poil » utilisé par les colons. Il semblerait que ce « à poil » soit la traduction hispanisante du tirumbae noté par Cardús (1886, p. 269) ou du itiru coimbae utilisé dans l'Isoso : tiru étant la tunique traditionnelle des hommes chiriguano, le terme voudrait dire « sans vêtements » (ce qui aurait été traduit par « à poil »), signalant, par là aussi, ce caractère de sauvage (Combès 2004). Enfin, sur le front oriental du Chaco, les actuels Ayoré étaient connus sous le nom générique de Moro. Ce terme chamacoco a un sens proche de «ennemi», au sens générique (il est utilisé indistinctement pour désigner différents groupes, aussi bien ayoré que sanapaná par exemple). L'armée paraguayenne reprit le terme de ses guides chamacoco et le diffusa largement dans la région lors de la guerre du Chaco. À propos du terme " Chamacoco », disons simplement qu'il est employé dans l'une des langues qui couvraient l'arc septentrional du Chaco (gorgotoqui, chiquito ou arawak) et, plus probablement, en chané-arawak. Il désignait de façon générique les populations de l'intérieur du Chaco et voudrait dire quelque chose comme «les chiens» (Richard 2008a). À l'intérieur de l'espace chamacoco, nous retrouverons aussi des « voleurs » (horio), des « sauvages » ou des « épars» (tomaraho) ou des « déchets » (ebytoso). Sur la frange orientale du Chaco, nous trouvons aussi des « galeux » (guaykuru), des « esclaves » (niyolola) ou des « ceux-avec-qui-on-senettoie-le-cul » (dukchént dotéer) (Cordeu 1981).

Ces quelques exemples suffisent à montrer le sens et l'identité d'ensemble de ce passage par lequel, en quelques décennies, les « avec-qui-on-se-nettoie-le-cul », les « merdes froides », les « chiens », les " gueules d'iguane», les « esclaves », 
« trop esclaves » ou « petits esclaves », les « sans vêtements » et autres « voleurs » et « sauvages » sont unanimement venus à s'appeler « les hommes », « les gens » ou « les personnes ». La carte ci-dessous permet de se faire une représentation d'ensemble de ce basculement (Figure 2).

Plusieurs éléments doivent attirer notre attention, car ces deux cartes ne sont pas seulement différentes quant à l'identité des noms retenus, mais aussi, et plus fondamentalement, quant à la façon dont ils se produisent et s'organisent. Nous passons ainsi d'un système de termes à caractère adjectivant à un autre à caractère substantivant. « Esclaves », « voleurs », " sauvages », etc., adjectivent l'autre et qualifient une relation. Nivaclé, Ayoré, Ishir, etc., substantivent le soi en le soustrayant à ces relations. Dans le premier cas, ce n'est pas un terme qui est nommé, mais une relation à ce terme. Car, lorsqu'on dit de quelqu'un qu'il est un « serf » ou un « esclave », on est en train de le définir par rapport à une relation de servitude. C'est cette relation qui est nommée et non point quelque particularité intrinsèque à l'un de ses termes. Ce qui explique comment, en des lieux différents du Chaco, des groupes linguistiquement et culturellement hétérogènes sont venus à être identifiés sous les mêmes termes. Qu'ont en commun ainsi les Tapuy (« esclaves » en chiriguano) de la marche occidentale du Chaco et les Niyolola (« esclaves» en guaycuru) de la marche orientale, si ce n'est leur relation de subordination vis-à-vis des caciquats chiriguano dans un cas et des capitaineries guaycuru dans l'autre ? La même question peut être posée au sujet des « sauvages » (Lumnanás) et des « sauvages » (Tomaraho) que rien ne réunit, sauf leur situation périphérique vis-à-vis de ceux qui les nomment ainsi. C'est ainsi aussi que l'on trouvera différents types de Ñanaigua et de Kaiingua dans toute la périphérie de la sphère guaranophone sans que pour autant l'on puisse en déduire quoi que ce soit sur l'identité de ces différents groupes, etc. En traduisant des relations, ces termes permettent de dresser la carte ou le système des principaux types de rapports interethniques qui tissent cet espace en un moment déterminé. Ils servent moins à nourrir l'histoire particulière de tel groupe qu'à éclairer le champ relationnel au sein duquel il a pu, ou non, s'agencer historiquement.

Tandis que, dans la deuxième strate, chaque groupe a un nom, la première strate fonctionne selon un principe multi-nominatif. Dans cette strate, un groupe a une multiplicité de noms, chacun d'entre eux circonscrit à la relation d'énonciation spécifique dont il est le résultat. Dans la strate contemporaine, ce groupe se doit d'avoir un seul nom, un "vrai nom », transcendant et identique à lui-même quel que soit l'interlocuteur et les circonstances précises d'énonciation. À vrai dire, il n'y a pas seulement passage d'un principe multi-nominal à un autre uninominal, car celui-ci s'accompagne d'un réagencement objectif par lequel le groupe en vient à devenir un. Reprenons l'exemple nivaclé. Comme nous l'avons dit, les sources ne gardent pas de traces de ce terme avant les mentions contemporaines, dès la fin des années 1950 . Non seulement ce terme n'est pas mentionné, mais il n'y a pas, non plus, la mention d'un terme alternatif, exprimé en nivaclé, 


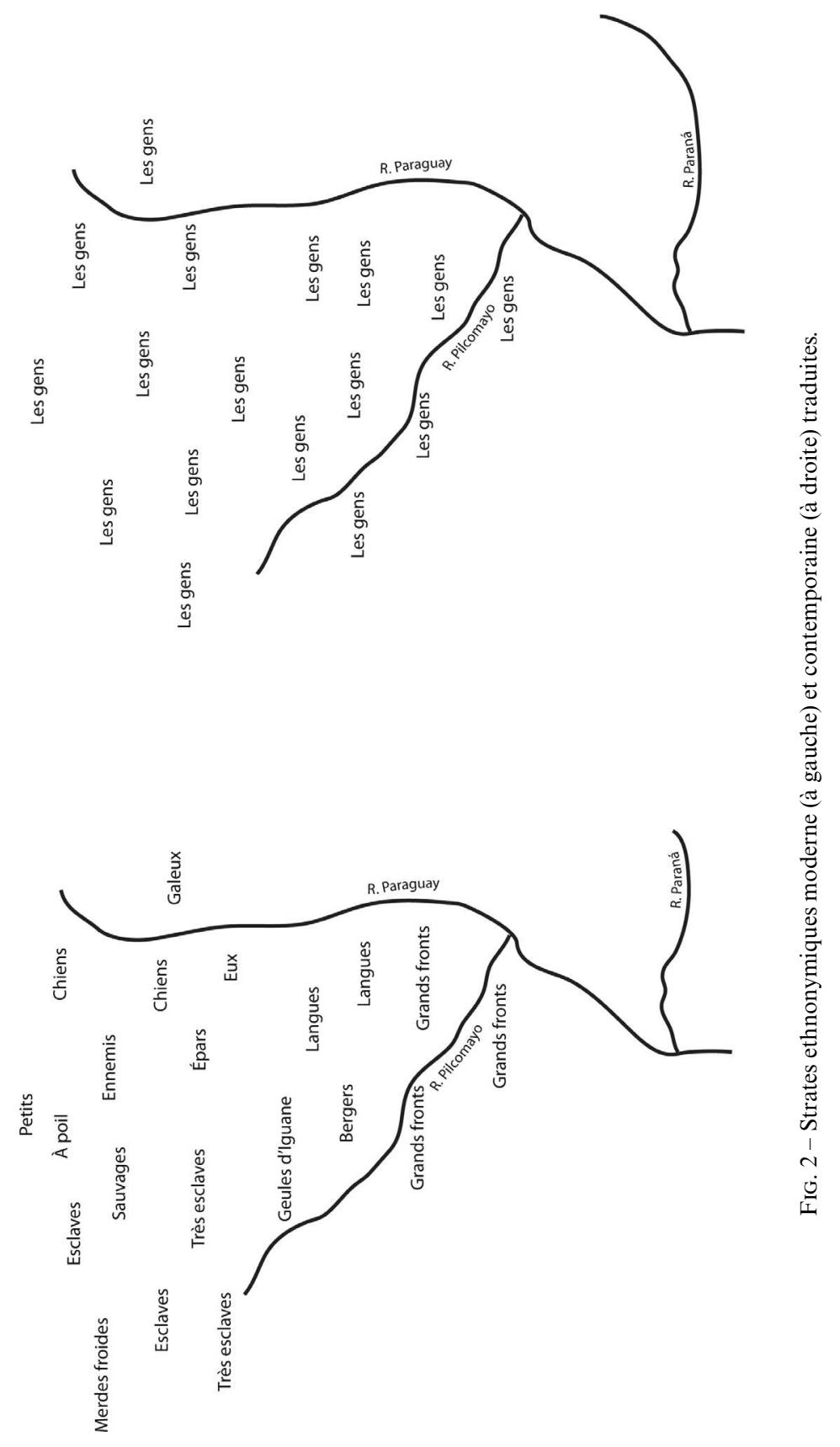


pour désigner l'ensemble de ces populations. Les campements les plus proches de la zone de contact maká sont dits wentusij (guentuse); ceux participant de la frontière lengua (enlhet) sont dits suhín; ceux situés à proximité de la ligne de contact chorote sont dits ashluslay, etc. Les campements sont dispersés, aucune forme de centralisation politique n'existe et les lignes d'affirmation identitaire s'organisent plutôt autour de formes politiques - chefferies, caciquats - que de critères ethniques ou linguistiques ${ }^{6}$. Lorsque la guerre du Chaco se prépare, un espion paraguayen peut alors communiquer à son état-major, alarmé, que les fortins boliviens du Pilcomayo comptent une multitude de tribus « amies » des Ashluslay, des Chulupí, des Suhín, etc. (Kehn 1929). Ce n’est que, lorsque ces populations ont été concentrées dans les missions catholiques de la zone, désarticulées de la trame politique indienne et encapsulées en un dispositif précis de gestion sociale et linguistique que l'unité du groupe en vint à être constituée. Ce n'est qu'alors, en tout cas, qu'un terme en nivaclé désignant quelque chose comme les Nivaclé fit surface. Le processus d'objectivation du terme répond ainsi, sur le plan nominal, à celui qui s'exerce sur le corps social concret.

Enfin, l'on passe d'un système de renvois croisés - chacun est nommé par autrui dans une langue qui n'est pas la sienne - à une collection d'énonciations autonomes - chacun se nomme lui-même dans la langue qui est la sienne. En conséquence, dans la strate déchue, l'opération d'identification est toujours médiatisée par un groupe tiers et comporte au moins deux articulations - A utilise le nom que $\mathrm{B}$ donne au groupe $\mathrm{C}$. Cette relation est donc indirecte et comporte au moins trois termes. Par contre, dans la strate émergente, cette identification se veut immédiate et non articulée, chacun se donnant soi-même un nom. Dans le premier cas, ces noms connectent des instances différentes - celui qui dénomme, celui qui est nommé, etc. - et tissent un espace articulé par différentes chaînes de nomination. Ces chaînes dessinent, nous le verrons, comme le canevas de fond de l'espace sociopolitique du Chaco. Elles lui impriment un caractère, elles organisent un «motif ». Aussi, si l'on cherchait à comprendre ce motif, il conviendrait de ne pas défaire le tissu pour étudier chaque fibre séparément. Il s'agirait, au contraire, de lire la trame qui s'en dégage, le texte bigarré de cette conversation intime qui anime le Chaco dans son ensemble. Il faudrait saisir cette discussion dans laquelle s'invitent les «avec-qui-on-se-nettoie-le-cul», les «gueules d'iguane », les «petits esclaves» et les «merdes froides». Or le grand peigne ethnologique a démêlé tout cela. Il a installé ce silence désolant, ces humanités parallèles qui chuchotent des mots autistes, chacune dans son coin, autour de son feu. Une couche épaisse d'interprétations et de déductions de tous ordres est venue conforter cette «évidence ». Il faudrait la désamorcer. Il faudrait pouvoir montrer comment l'occupation et la colonisation définitives du Chaco sont venues se signifier à travers l'institution unanime de l'ethnonyme «les hommes». 


\section{STRATES ETHNONYMIQUES}

Une césure profonde sépare donc ce que nous appelions les strates ethnonymiques "moderne » et "contemporaine ». Or cette discontinuité n'est pas la première en date et, dès qu'on observe cet espace dans la durée, d'autres failles historiques apparaissent qui viennent interrompre la trace nominale des populations indiennes du Chaco. Ces failles organisent historiquement cet espace comme une succession de strates consistantes en elles-mêmes, mais discontinues entre elles. En effet, à la différence d'autres régions du continent, le Chaco fut l'objet de projets successifs d'occupation et de colonisation (les expéditions espagnoles du XVI ${ }^{\mathrm{e}}$ siècle, l'offensive jésuite au XVIII ${ }^{\mathrm{e}}$ siècle, les avancées du front pionnier au XIX ${ }^{\mathrm{e}}$ siècle, le déploiement militaire lors de la guerre du Chaco...) qui ne réussirent pas à s'installer dans la durée, qui furent vite recouverts par la brousse et que d'importants hiatus historiographiques séparent. Chacune de ces avancées apporta son lot de descriptions et ses cartes ethniques (Lowrey 2006). Or il apparaît que ces cartes, tout comme celles que l'on vient d'analyser, sont irréconciliables entre elles. L’ensemble des "générations » identifiées au $\mathrm{XVI}^{\mathrm{e}}$ siècle ne garde pas de relation avec celui des " nations » décrites par les jésuites au XVIII ${ }^{\mathrm{e}}$ siècle, ni avec les « tribus » signalées au XIX ${ }^{\mathrm{e}}$ siècle, ni encore avec les groupes actuels. Comme si, dans chaque strate, cet espace social avait été entièrement réécrit de sorte que, en général, les entités identifiées dans une strate ne survivent pas dans la suivante.

Nous dirons donc qu'il y a émergence d'une « strate ethnonymique » lorsque l'ensemble des dénominations ethniques retenues dans les sources change. De façon générale, l'ethnologie du Chaco a tenté de construire des généalogies capables de traverser ces failles en continuité. L'on dira ainsi que les Ayoré actuels sont les Guarañoca du début $\mathrm{du} \mathrm{xx}^{\mathrm{e}}$ siècle, eux-mêmes issus des Zamuco du XVIII $^{\mathrm{e}}$ siècle, etc. (Bremen $\left.1991 ; 2005\right)$. Ce procédé efface la discontinuité des noms pour mieux affirmer la pérennité des groupes ethniques concernés. Pour nous, il s'agit, au contraire, de montrer comment la discontinuité du champ nominal correspond à un bouleversement profond dans le champ relationnel qui organise cet espace. Ces noms traduisent, en effet, une certaine disposition à la guerre, à des formes de captivité, à des alliances et à des formes de dépendance, des asymétries et des équivalences qui marquent les relations inter-groupales dans le Chaco. Dans notre hypothèse, les césures entre les strates nominales correspondent à des moments denses où ces relations - la guerre, la captivité, les alliances, etc. - sont bouleversées. Or ces analyses ne peuvent être menées qu'à la condition de respecter la consistance des strates ethnonymiques et de rendre visible - et donc problématique - leur discontinuité historique. Nous pourrons alors passer d'une compréhension généalogique (toutes ces familles, ces descendants et ces ancêtres qui peuplent l'ethnologie du Chaco) vers une autre, relationnelle, des dynamiques indiennes. 
L'approche généalogique imagine une discontinuité sociologique sur fond de continuité historique. Elle pense le Chaco comme un système d'entités sociales étanches - les Ayoré, les Chamacoco, les Nivaclé, etc. - traversant en toute continuité les failles de l'histoire. Or il nous semble que nous apprenons plus du paysage social chaquéen du $\mathrm{XIX}^{\mathrm{e}}$ siècle en sachant, par exemple, que le nom "Chamacoco" émerge au même moment que "Caduvéo " (ou que " Guaycuru » décline en même temps que « Chiriguano »), qu'en s'accrochant désespérément à la plus infime des preuves (une ressemblance phonétique, une coïncidence géographique) pour affirmer sa continuité généalogique avec les « Zamuco » du XVIII ${ }^{\mathrm{e}}$ siècle ou avec les « Tamacoci » du XVI ${ }^{\mathrm{e}}$ siècle. Il nous semble en ce sens aussi que l'on ne peut pas comprendre de façon adéquate l'agencement historique ni même les spécificités sociologiques des groupes chamacoco sans les réintroduire dans la sociologie générale de cet espace.

Il est donc tout à fait insuffisant d'écrire l'histoire particulière des Ayoré, des Nivaclé ou des Chamacoco. Non pas que l'on puisse simplement mésestimer cette démarche - elle a orienté nombre de très bon travaux sans lesquels nous ne pourrions pas écrire aujourd'hui -, mais elle risque d'écarter cela même qui fait l'intérêt et la spécificité ethnologique de cette région, à savoir, l'extrême malléabilité de ses formations sociales, l'extrême rapidité des processus de recomposition et de réagencement ethnique, cette facilité hallucinante avec laquelle des gens de langues et d'horizons différents en viennent à se mixer, à se décomposer et à se recomposer dans des laps historiques extrêmement brefs. Quelle est la profondeur historique des formations ethniques du Chaco ? Voici une question fondamentale des problématiques chaquéennes que les apôtres des humanités parallèles et des " peuples isolés » sont dans l'incapacité même de formuler. Comment tel groupe est-il venu à se constituer? Pour s'inscrire dans quelle durée ? À travers quels mécanismes ? Que se passe-t-il lorsqu'il vient à se désintégrer ? Voilà des problèmes que le primat téléologique de la linguistique et de sa machine généalogique a empêché de se poser.

\section{Chaînes de NOMINATION}

Une fois le principe "stratigraphique » posé, le problème reste de savoir comment travailler, à l'intérieur d'une strate déterminée, avec les différents systèmes de noms qui l'organisent. Or nous savons qu'en règle générale, ces noms ne sont jamais auto-attribués - il n'y a pas de terme en chulupí pour nommer les Chulupí, il n'y en a pas, non plus, en chamacoco pour nommer les Chamacoco, etc. Ou encore, inversement, nous savons que ces termes sont toujours extérieurement attribués et qu'ils le sont en général dans une langue tierce, c'est-à-dire qu'ils supposent au moins deux entités et qu'ils traduisent leur relation. Il est donc possible de restituer des connections entre ces termes et de les resituer en des 
enchaînements. Ces enchaînements permettent de coordonner la strate en question et de saisir ses principales articulations.

Prenons par exemple les chaînes de dénominations qui caractérisent les marges orientale et occidentale du Chaco. Du côté occidental, aux frontières de l'espace colonial espagnol, le paysage est dominé par le terme « chiriguano » qui disparaît vers la fin du XIX ${ }^{\mathrm{e}}$ siècle (Combès et Saignes 1991; Pifarré 1989 ; Combès 2005). Nous avons vu que l'étymologie du terme a fait l'objet de plusieurs hypothèses (Combès 2010, p. 129 sq.), mais celles-ci coïncident quan t à son caractère exogène - «chiriguano » n'étant pas un terme de langue chiriguano. Plus à l'intérieur du Chaco, nous trouverons des Tapuy. Il s'agit d'un terme guarani (chiriguano) utilisé pour désigner des populations - Chané, Isoceño, etc. - initialement non guaranophones. Encore plus à l'intérieur, il y aura des Tapieté, qui est aussi un terme guarani désignant des populations vraisemblablement d'horizon zamuco ou mataco (Combès 2008). Enfin, en approchant le centre du Chaco, il y aura toutes sortes de Yanaigua, Sirakúa et autres Empelotas fragmentairement mentionnés dans les sources. Soit :

Espace colonial $=>$ Chiriguano $=>$ Tapuy $=>$ Tapieté $=>$ Yanáigua, Sirakúa, etc.

Du côté oriental du Chaco nous trouverons d'abord des Guaycuru bien connus à l'époque coloniale et dont l'ethnonyme s'efface aussi quelque part au milieu du XIX ${ }^{\mathrm{e}}$ siècle. " Guaycuru » et le terme alternatif de « Mbayá » sont des termes guarani utilisés pour désigner des populations non guaranophones situées au-delà de la frontière coloniale paraguayenne. Vers l'intérieur du Chaco, nous trouvons des Niyolola, terme guaycuru utilisé pour nommer des populations d'horizon arawak (Guaná, Terena, etc.) (Sánchez Labrador 1910). Nous trouverons ensuite des Chamacoco, terme vraisemblablement arawak (Terena, Guaná) utilisé pour désigner des populations d'horizon zamuco venues de l'intérieur (Richard 2008a ; Combès 2009). Plus loin nous trouverons des Moro et des Tomaraho, qui sont des termes chamacoco désignant les populations les plus reculées vers l'intérieur du Chaco (Richard 2008a et b). Soit :

Espace colonial $=>$ Guaycuru $=>$ Niyolola $=>$ Chamacoco $=>$ Moro, Tomaraho, etc.

Ces deux enchaînements sont homologues et se construisent sur une même série d'articulations $\left(1^{\mathrm{e}}, 2^{\mathrm{e}}, 3^{\mathrm{e}}\right.$, etc.). Soit :

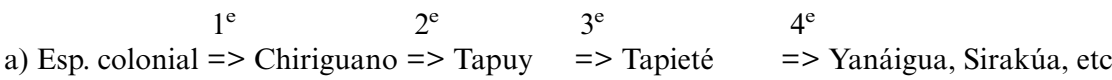

b) Esp. colonial $=>$ Guaycuru $=>$ Niyolola $=>$ Chamacoco $=>$ Moro, Tomaraho, etc.

L'identité entre ces deux enchaînements et la nature des articulations qui les structurent apparaissent plus clairement si l'on traduit les termes qui les composent. Nous ne pouvons pas fournir ici le détail de cette démonstration (Richard 2008a, chap. III), mais si l'on restitue le sens des termes enchaînés, le résultat approximatif est : 


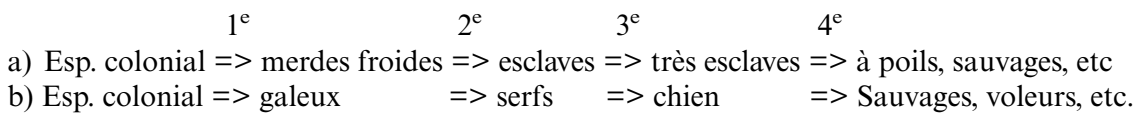

Partant des deux marges opposées du Chaco, ces enchaînements dessinent comme la trame ou le canevas de fond de cet espace. Celui-ci tient moins à l'identité des termes qu'à leur position relative dans le système de coordonnées que ces articulations dessinent. Il en est ainsi de « Tapieté » et « Chamacoco ». Sur le plan ethnographique, rien ne relie ces deux groupes qui sont de langue et de culture différentes et qui habitent les marges opposées du Chaco. Nonobstant, ils se situent tous deux dans une même position relative à l'intérieur des enchaînements qui organisent cette strate. Dans notre hypothèse, de par cette homologie et au-delà de leurs différences ethnographiques, ces deux formations sociales sont confrontées à un même type de problèmes sociologiques (dépendance périphérique vis-à-vis des grands caciquats de la frontière, flux asymétrique de captifs, impossibilité de se concentrer géographiquement, etc.) et la solution que chacune d'entre elles offre à ces problèmes doit être analysée par rapport à celle qu'offre l'autre. Il y a donc des problèmes sociologiques qui sont spécifiques à chacune de ces articulations et qui se posent en des termes homologues pour chacune des populations - quelle que soit leur langue - situées en une même position corrélative.

La première articulation correspond à la frontière coloniale. Il s'agit sans doute du cas de figure le plus étudié : la « frontière chiriguano » ou la « frontière guaycuru ", tout comme la "frontière mapuche» au sud du Chili et dans la Pampa, ont eu un rôle structurant dans l'imaginaire et dans la physionomie des sociétés coloniales. Il s'agit aussi du cas de figure le plus documenté dans les archives et dont les analyses historiques ont été les plus poussées. Elles dessinent une typologie précise - celle des sociétés indiennes « de la frontière » - qui pose des problèmes spécifiques : intensification et redéfinition de l'activité guerrière, formation de grands caciquats, concentration économique et politique, expansion territoriale, rôle social accru des métis, transferts et circulations techniques et idéologiques, formes juridiques et politiques de la relation frontalière, etc. ${ }^{7}$.

La deuxième articulation correspond à la relation tributaire qu'entretiennent les grands caciquats chiriguano et guaycuru avec les foyers de peuplement arawak (Chané, Guaná, Terena) situés dans leur arrière-pays. Les termes tapuy et niyolola, qui furent couramment traduits à l'époque coloniale par « esclaves » ou « serfs », montrent bien la nature de cette relation. Or elle s'organise différemment de chaque côté du Chaco. La mainmise guarani sur le substrat paysan chané s'est soldée, du côté occidental, par une " guaranisation » linguistique poussée des populations indigènes (Combès et Saignes 1991 ; Combès 2005). Le contrôle guaycuru sur les foyers de peuplement guaná et terena dans le Haut Paraguay, qui a une profondeur historique équivalente, n'a pas produit de " guaycuruisation » ni linguistique, ni politique des sociétés concernées (Cardoso de Oliveira 1960). 
Dans le premier cas, l'assujettissement des populations chané s'exerce sur place à travers un processus accéléré de métissage social. Dans le deuxième, les « capitaines » guaycuru contrôlent les villages guaná à distance tout en affichant une bien connue répugnance au métissage. Ce ne sont là que quelques éléments qui permettent d'entrevoir le type de problèmes que pose cette deuxième articulation : quelle gestion de la donne linguistique ? quelles formes d'inclusion ou d'articulation sociale pour les populations assujetties? quels processus précis d'ethnogenèse en résultent ? à travers quelles solutions symboliques ou idéologiques ? etc.

La troisième articulation correspond à ce que Susnik (1981) théorisa comme situation de «dépendance périphérique ». Elle caractérise des populations non encore entièrement engrenées dans l'orbite tributaire, mais déjà en situation de dépendance relative vis-à-vis d'elle, que les termes « chiens » ou « très esclaves » traduisent bien ${ }^{8}$. De notre point de vue, il s'agit là d'une articulation décisive pour comprendre la physionomie et la dynamique indienne du Chaco. L'articulation tributaire s'organise sur les marches du Chaco et ne le concerne qu'indirectement. L'articulation de « dépendance périphérique » travaille, en revanche, à l'intérieur du Chaco et explique la formation de bon nombre de ses groupes ethniques. Ainsi, par exemple, elle est décisive pour comprendre la nucléation, à un moment déterminé (au cours du XVIII ${ }^{\mathrm{e}}$ siècle), de ceux qui seront plus tard objectivés sous l'étiquette "Chamacoco » (Richard 2008a; Combès 2009). Le même constat peut être fait au sujet des Tapieté chaquéens (Combès 2008), mais aussi des groupes de famille mascoy - les « faux Guaná » de Métraux (1946b) dont la situation mi-indépendante, mi-périphérique vis-à-vis des grands foyers guaná du Haut Paraguay est déjà décrite dans les sources du XVIII ${ }^{\mathrm{e}}$ siècle (Azara 2009). Il nous semble, ici aussi, que cette articulation suppose des problèmes spécifiques dans la solution desquels ces différentes sociétés se sont construites. Fondamentalement, celui de gérer une situation hypostatique, à la marge des grands foyers tributaires, tiraillée entre les forces d'attraction et d'expulsion que ces foyers installent. Sur la marge orientale du Chaco, la fête des anabsoro, rituel pratiqué par les populations chamacoco, mais aussi par les populations mascoy plus méridionales, est particulièrement éclairante à ce sujet: elle explique l'avènement des «hommes nouveaux" (les Chamacoco), comme le résultat d'une période contradictoire où les « hommes anciens », venus de la brousse, s'installent dans la périphérie riche et abondante des "étrangers furieux », qui leur apprennent la peinture corporelle, le tissage, les chants et l'intelligence, mais qui « mangent» leurs enfants et leurs femmes et les obligent in fine à une longue guerre d'émancipation qui sera aussi celle de leur affirmation sociale (Escobar 1999 ; Cordeu 2003 ; Susnik 1969).

Enfin, plus au centre du Chaco, la quatrième articulation marque le seuil ultime de l'action concentratrice des caciquats de la frontière. Au-delà, des populations démographiquement plus réduites et territorialement plus éparses 
- Tomaraha, Manjui, Ayoré... - peuplent l'intérieur de la région. Elles se placent dans une situation d'extériorité vis-à-vis des circuits de captivité. En tout cas, elles constituent leur seuil purement négatif : il s'agit de populations qui fournissent des captifs sans pour autant pratiquer la captivité. Toute une série de problèmes - de démographie, de conception et de fonction de la guerre, de définition de l'ennemi, de hiérarchisation sociale, etc. - en découlent. Si la captivité joue un rôle de premier ordre dans le fonctionnement des autres sociétés du Chaco, celles-ci pourraient être définies par son absence. C'est ainsi, par exemple, que, si l'on compare les univers symboliques tomaraho, ayoré et chamacoco, à conditions culturelles et sociologiques égales (un même tronc linguistique, une même organisation clanique issue d'anciennes colonisations otuqé-bororó, un même calendrier rituel mettant en scène les mêmes personnages, une culture technique commune, etc.), un des éléments les plus fortement clivants tient à la place accordée au problème du captif : l'univers idéologique chamacoco est entièrement travaillé, occupé, préoccupé par la question de la captivité ; celui des Tomaraho ou des Ayoré semble s'organiser sur son absence, c'est-à-dire, sur le problème inverse de l'élimination de l'autre (D'Onofrio 2003).

Ces enchaînements nominaux permettent donc d'identifier une série d'articulations paradigmatiques qui jalonnent le paysage social du Chaco. Ils traduisent la morphologie des transitions et des médiations qui modulent cet espace. Ils dessinent des schémas de connexions. Ils permettent donc de construire une typologie des rapports politiques et interethniques qui caractérisent une strate ethnonymique déterminée. À travers ces chaînes, on pourra coordonner la strate et identifier des situations caractéristiques de la première, deuxième ou troisième articulation. On pourra alors penser la situation de telle ou telle formation sociale non plus en la référant généalogiquement à sa propre histoire, qui est toujours l'histoire de sa permanence, mais au champ dynamique des relations qui structurent cet espace. Ce ne sont pas des corps formés ou donnés qui nous intéressent ici, mais bien le système de relations au sein duquel, par la dynamique qu'il impose, ceux-ci ont pu ou non se former à un moment déterminé.

\section{CONCLUSION}

Entamé dans les décennies qui suivent la guerre du Chaco (1932-1935), le bouleversement de l'ethnonymie chaquéenne et l'émergence d'une nouvelle strate de «noms ethniques » correspond à la désactivation du champ de relations interethniques, au démantèlement de l'espace politique indien et à l'occupation et colonisation définitive de cet espace. Ce n'est que lorsque ces populations, fortement décimées par l'occupation militaire, les exactions et les épidémies, ont été regroupées en réserves ou en missions que leur " nouveau nom » a émergé. Les différents systèmes d'adjectivations et de dénominations croisées sont alors 
neutralisés et cèdent, face à des termes génériques et communs à l'ensemble - « les hommes »-qui perdent progressivement leur force distinctive à l'intérieur du monde indien.

Or, dès qu'on se concentre sur la strate nominative antérieure et qu'on restitue les connexions et les renvois croisés qui la fondent, une image d'ensemble se forme qui rend plus intelligible le fonctionnement de cet espace tout en permettant d'identifier les principales articulations et types de rapports interethniques qui l'organisent. Nous avons ainsi essayé de montrer comment, en connectant les noms ethniques aujourd'hui déchus, il est possible d'identifier les «chaînes nominatives » qui tissent et paramètrent une strate historique déterminée. À travers ces chaînes, il est possible d'identifier des articulations paradigmatiques qui posent des problèmes spécifiques d'organisation sociale. Le Chaco boréal apparaît alors comme un espace organisé depuis sa périphérie vers son centre, à travers quatre articulations principales : a) frontière coloniale, b) relation tributaire, c) relation de dépendance périphérique, d) relation d'extériorité. Il s'agirait donc d'étudier cette région en reflétant les unes sur les autres les populations situées en une même position et confrontées par conséquent à un même type de problèmes. De ce point de vue, la proximité linguistique n'est pas un critère immédiatement pertinent pour relier les différents dossiers : on en apprendra plus sur les Chamacoco en les mettant en regard avec les Tapieté, qui parlent une autre langue et vivent de l'autre côté du Chaco, mais qui se situent dans une même position corrélative, qu'en les référant aux Ayoré, de même tronc linguistique et géographiquement voisins, mais dont l'articulation à l'espace général du Chaco est autrement médiatisée et construite.

Le bouleversement massif de l'ethnonymie du Chaco dans les dernières décennies a été reçu comme allant de soi. Vidé de toute signification, naturalisé dans son avènement et son déploiement historique, il est devenu un "nonévénement ». Mais alors, dans ce chœur de soliloques où chacun se dit lui-même et où chaque groupe est celui des " Hommes », dans cette vitrine humaniste où personne n'aurait plus besoin de nommer l'autre puisque chacun se nomme lui-même dans la case qui est la sienne, le nom et l'ethnonyme n'auraient-ils pas changé de nature et de fonction? Ne seraient-ils pas en train de nommer non seulement autrement mais encore autre chose? Le changement du nom, de sa nature et de sa fonction, ne signalerait-il pas, non pas la permanence d'une identité qui se dirait autrement, mais au contraire une transformation profonde dans l'économie même des identités et dans l'opération de (se) nommer ? Degré zéro d'un nom que celui qu'on s'attribue soi-même pour signifier la plus banale et générale des catégories - mais il y a quelque chose de profondément émouvant en ce geste : nous sommes... « des personnes ». Quelqu'un en doutait ?*

* Manuscrit reçu en mars 2010, accepté pour publication en août 2011. 


\section{Notes}

1. Cet article est une réécriture du premier chapitre de ma thèse doctorale (Richard 2008a).

2. Observation personnelle et voir aussi Grünberg et Grünberg (1975), Zanardini et Biedermann (2001).

3. "Los ayoreo se denominan a sí mismos ayoreode, que es la forma plural de ayoréi, el hombre, cuyo femenino es ayoré» (Bórmida et Califano 2003, p. 19; traduction en français dans le texte de l'auteur, N. R.)

4. Voir le Diccionario guarani-español (2000) et, aussi, Susnik et Chase-Sardi (1995).

5. L'hypothèse de Combès et Saignes (1991) est lumineuse et il nous semble qu'elle a eu le mérite d'introduire dans le « dossier chiriguano », jusqu'alors trop souvent envisagé depuis sa seule articulation aux plateaux andins, une profondeur et une problématique chaquéennes. Elle n'est pas pour autant exempte de problèmes. D'abord, parce que le nom serait construit à partir de termes appartenant à des langues différentes - qui donc est celui qui parle ? ; ensuite, parce que la pertinence du terme « Guaná » n'est aucunement évidente : il faudrait expliquer pourquoi un nom construit et utilisé dans le piémont bolivien - Chiriguaná - aurait recours à un autre - Guaná - qui apparaît plus tard dans les sources et dont l'usage est circonscrit au Haut Paraguay, à un millier de kilomètres de distance.

6. Telle est l'hypothèse de base pour la clarification des taxonomies ethniques entreprise, pour la zone du Pilcomayo, par la série de travaux dirigée par Braunstein (1990-2005).

7. Voir, entre autres, Obregón, Capdevila et Richard (2011). Notons d'ailleurs que ces « frontières » s'organisent selon une même stratigraphie ethnonymique : le terme « Mapuche » émerge quelque part au début du XviII ${ }^{\mathrm{e}}$ siècle (Boccara 1998 ; Zavala 2000), au moment même où l'on passe de "Chiriguanae » à " Chiriguano » sur le versant occidental du Chaco (Combès et Saignes 1991) et de « Guaycuru » à « Mbayá » sur son versant oriental (Cardoso de Oliveira 1960).

8. L'utilisation de la catégorie «chien » dans les taxonomies indiennes est problématique et il faudrait, bien sûr, la préciser ethnographiquement dans chaque cas. Nordenkiöld (1912) notait déjà avec stupeur la quantité de chiens présents dans les campements indiens, ainsi que la violence avec laquelle ils étaient traités. Villar (2005) a analysé cette position inter-moyenne du chien dans les sociétés indiennes du Chaco : il est maltraité, marginalisé et vit dans l'abandon. Mais il est omniprésent, il reçoit un nom propre et s'inscrit dans les territorialités - familiales, politiques - du village. Il vit dans sa périphérie, ni complètement intégré, ni entièrement expulsé.

\section{RÉFÉRENCES CITÉES}

Azara Félix d'

2009 Voyages dans l'Amérique méridionale 1781-1801, présenté et édité par Nicolas Richard, Presses universitaires de Rennes/CoLibris, Rennes [1809].

BALDUS Herbert

1927 «Os Indios chamacocos e a sua lingua », Revista do Museu Paulista, 15 (2), pp. 5-68.

BÉLAIEFF Juan

1941 Mapa etnográfico del Chaco Paraguayo, Sociedad Científica del Paraguay, Asunción [1932].

BERNAND Carmen

1977 Les Ayoré du Chaco septentrional : étude critique à partir des notes de Lucien Sebag, Mouton, Paris. 
BLASER Mario

2004 "Way of life" or "who decides": development, Paraguayan indigenism and the Yshiro people's life projects », in Mario Blaser, Harvey Feit et Glenn McRae (éd.), In the way of development: Indigenous peoples, life projects and globalization, Zed Books, Londres.

BOCCARA Guillaume

1998 Guerre et ethnogénèse mapuche dans le Chili colonial : l'invention du soi, L'Harmattan, Paris.

BogGiani Guido

1894 «I Ciamacoco», Atti della Societa Romana di Antropologia, 2 (1), pp. 9-127, Roma

BóRmida Marcelo et Mario CALIFAno

2003 «Los ayoreo del Chaco boreal », in José Zanardini (éd.), Cultura del pueblo ayoreo, Centro social indígena/Centro de estudios antropológicos de la universidad Católica, Asunción, pp. 7-240 [1978].

Bossert Federico et Alejandra SiFFredi

2011 «Las relaciones interétnicas en el Pilcomayo medio : la guerra indígena y sus transformaciones (1882-1938) », Población y Sociedad. Revista regional de Estudios Sociales, 18 (1), pp. 3-48

BRAUNSTEIN José

1990-2005 Hacia una nueva carta étnica del Chaco, 8 vol., CONICET, Las Lomitas, Argentine.

BREMEN Volker von

$1991 \quad Z$ wischen Anpassung und Aneignung: Zur Problematik von WildbeuterGesellschaften im modernen Weltsystem am Beispiel der Ayoréode, Anacon, Munchen.

2005 «Impactos de la guerra del Chaco en la territorialidad ayorea » in Nicolas Richard, Luc Capdevila et Capucine Boidin (éd.), Les guerres du Paraguay aux XIX ${ }^{e}$ et $X X^{e}$ siècles, CoLibris, Paris, pp. 263-280.

Cardoso de Oliveira Roberto

1960 Do índio ao bugre : o processo de assimilação dos Terêna, Livraria Francisco Alves, Rio de Janeiro.

CARDús José

1886 Las Misiones franciscanas entre los infieles de Bolivia : descripción del estado de ellas en 1883 y 1884, Librería de la Inmaculada Concepción, Barcelona.

Chamorro Andrea

2006 Lumnanas (gente del monte) : los manjui, Ceaduc, Asunción.

CHASE-SARdi Miguel

2003 ; Palavai Nuu! Etnografia nivaclé, 2 vol., Ceaduc, Asunción.

ComBÈs Isabelle

2004 «Tras las huellas de los ñanaigua : de tapii, tapiete y otros salvajes en el Chaco boliviano », Bulletin de l'Institut français d'études andines, 33 (2), pp. 255-269. 
2005 Etno-historias del Isoso : chané y chiriguanos en el Chaco boliviano ( siglos XVI $a X X)$, Institut français d'études andines, La Paz.

2008 "Los fugitivos escondidos. Acerca del enigma tapiete», Bulletin de l'Institut français d'études andines, 37 (3), pp. 511-533.

2009 Zamucos, Instituto latinoamericano de misionología, Cochabamba, Bolivie.

2010 Diccionario étnico : Santa Cruz la Vieja y su entorno en el siglo XVI, Instituto Latinoamericano de Misionología, coll. «Scripta Autochtona » 4, Cochabamba, Bolivie.

Combès Isabelle et Thierry SAIGNES

1991 Alter Ego : naissance de l'identité chiriguano, École des hautes études en sciences sociales, coll. « Cahiers de l'Homme » 30 (nouvelle série), Paris.

CORDEU Edgardo

1974 «La idea de "mito" en las expresiones narrativas de los indios chamacoco o ishir », Scripta Ethnologica, 2 (2), pp. 75-117.

1981 Aishtuwénte : las ideas de deidades en la religiosidad chamacoco, tesis doctoral en Filosofía y Letras, Universidad de Buenos Aires, Buenos Aires.

2003 Transfiguraciones simbólicas : ciclo ritual de los indios tomaráxo del Chaco Boreal, Centro de Artes Visuales/Museo del Barro, Asunción [1999].

D'ONOFRIO Salvatore

2003 "Guerre et récit chez les Indiens ayorés du Chaco boréal paraguayen », Journal de la Société des Américanistes, 89 (1), pp. 39-81.

Diccionario guarani-español

2000 Instituto superior de Lenguas, universidad de Asunción, Asunción.

EsCOBAR Ticio

1999 La maldición de Nemur : acerca del arte, el mito y el ritual de los indígenas ishir del Gran Chaco paraguayo, Centro de Artes Visuales/Museo del Barro, Asunción.

FABRE Alain

2005-2007 «Los pueblos indígenas del Gran Chaco y sus lenguas », Suplemento Antropológico, Ceaduc, Asunción [4 parties : 1. "Los enlhet-enenlhet », 2005, 40 (1), pp. 503-569 ; 2. « Los mataguayo », 2005, 40 (2), pp. 313-435; 3. « Los guaykurú », 2006, 41 (2), pp. 7-131; 4. « Los zamuco», 2007, 42 (1), pp. 271-323].

GRUBB Warbroke

1911 An unknown people in an unknown land, Seeley, Londres.

GRÜNBERG Georg et Friedl GRÜNBERG

1975 Los chiriguanos, guarani occidentales del Chaco central paraguayo: fundamentos para una planificación de su desarrollo comunitario, Ceaduc, Asunción.

Hunt Richard

1915 El chorotí o yófuaha, H. Young, Liverpool. 
HAEKel Joseph

1955 «Berichte: Studienreise eines jungen Österreichers in Ostbolivien », Wiener Völkerkundliche Mitteilungen, 3 (1), pp. 100-103, Wien.

KeHN Roberto

1929 Memoria sobre la situación militar actual en la zona sudeste del «Chaco boliviano »: Media luna (Argentina), 26 de abril de 1929, Archivo del Estado Mayor Paraguayo, Asunción.

Kelm Heinz

1960 «Zur Frage der ethnographischen Einordnung der Ayoré, Moro und Yanaígua im Ostbolivianischen Tiefland », Baessler-Archiv, 8, pp. 335-361, Berlin.

LOWREY Kathleen

2006 «Entre estructura e historia: el Chaco », in Isabelle Combès (éd.), Definiciones étnicas, organización social y estrategias políticas en el Chaco y la Chiquitania, IFEA/SNV, Santa Cruz de la Sierra, Bolivie, pp. 25-31.

MÉtraux Alfred

1946a «Tribes of the Gran Chaco: present day locations (map) », in Julian H. Steward (éd.), Handbook of South American Indians, Smithsonian Institution, Bureau of American Ethnology, Washington, p. 204.

1946b "Ethnography of the Chaco », in Julian H. Steward (éd.), Handbook of South American Indians. 1. The marginal tribes, Smithsonian Institution, Bureau of American Ethnology, Washington, pp. 197-370.

New Tribes Mission

1955 Ayoré Pedagogical Grammar, Cochabamba, Bolivie.

Nino Bernardino de

1912 Etnografia chiriguana, Argote, La Paz.

NoRDENSKIOLD Erlan

1912 «La vie des Indiens dans le Chaco (Amérique du sud)», Revue de Géographie, Hors série, 6, pp. 1-277, Paris.

1929 Analyse ethno-géographique de la culture matérielle de deux tribus indiennes du Gran Chaco, Genet, Paris.

ObRegón Jimena, Luc CAPDEvila et Nicolas Richard

2011 Les Indiens des frontières coloniales: Amérique australe, Presses universitaires de Rennes, coll. " $\mathrm{XVI}^{\mathrm{e}}$ siècle/temps présent », Rennes.

PIFARRÉ Francisco

1989 Los guarani-chiriguanos. 2. Historia de un pueblo, Centro de Investigación y Promoción del Campesinado, La Paz.

RICHARD Nicolas

2008a Les chiens, les hommes et les étrangers furieux. Archéologie des médiations indiennes dans le Chaco boréal, 2 vol., thèse de doctorat en anthropologie sociale, École des hautes études en sciences sociales, Paris.

2008b "Los baqueanos de Belaieff », in José Braunstein et Norma Meichtry (éd.), Liderazgo, representatividad y control social en el Gran Chaco Sudamericano, Eudene, Buenos Aires, pp. 150-76. 
Rodrigues Do Prado Francisco

1795 «Historia dos Indios cavalleiros ou da naçâo Guaycurú », Revista do Instituto Histórico e Geográfico Brasileiro, 1, pp. 25-57, Rio de Janeiro [1839].

\section{Romano Santiago et Hernán CATtunaR}

1916 Diccionario chiriguanolespañol y españollchiriguano, Apostólico Colegio de Santa María de los Ángeles, Tarija, Bolivie.

SÁNCHEZ LABRADOR José

1910 El Paraguay católico, édité par Samuel Lafone Quevedo, Imprenta de Coni hermanos, Buenos Aires, 2 vol. [1770].

SEBAG Lucien

1964 "Compte rendu de mission chez les Indiens Ayoré du Paraguay et Bolivie », L'Homme, 4 (2), pp. 126-129

1965 « Le chamanisme Ayoreo », L'Homme, 5 (1-2), pp. 92-112.

SEQUERA Guillermo

2005 Chamacoco cosmografialCosmography chamacoco, Boras konsmuseum, Boras, Suède.

SifFREDI Alejandra et Susana SANTINI

1993 «Movimientos, localización y experiencia : una aproximación a la historia oral de los nivaclé septentrionales en los últimos sesenta años », Memoria Americana: Cuadernos de Etnohistoria, 2, pp. 1-29, Buenos Aires.

STERPIN Adriana

1993 "La chasse aux scalps chez les Nivacle du Gran Chaco », Journal de la Société des Américanistes, 79, pp. 33-66

SúSNIK Branislava

1957 Estudios chamacoco, Sociedad Científica del Paraguay y del Museo Dr. Andrés Barbero, coll. « Etnografía », Asunción.

1961 Apuntes de etnografia paraguaya, Museo Etnográfico Andrés Barbero, Asunción.

1969 Chamacocos I: cambio cultural, Museo Etnográfico Andrés Barbero, Asunción.

1978 Etnografia paraguaya (parte primera), Museo Etnográfico Andrés Barbero, Asunción.

1981 Etnohistoria de los chaqueños: 1650-1910, Museo Etnográfico Andrés Barbero, coll. « Los aborígenes del Paraguay » 3 (1), Asunción.

SúSNIK Branislava et Miguel Chase-SARDI

1995 Los Indios del Paraguay, Mapfre, Madrid.

UlRICH Mateo et Rosemary de UlRICH

2000 Dictionario ishiro-español, New Tribes Mission, Puerto Diana, Paraguay.

UNRUH Ernesto et Hannes KaLISCH

2003 "Enlhet-enenlhet. Una familia lingüística chaqueña », Thule. Rivista italiana di studi americanistici, 14-15, pp. 207-231. 
VILLAR Diego

2005 «Indios, blancos y perros », Anthropos, 100 (2), pp. 495-506, Freiburg.

ZANARdini José et Walter BIEDERMANN

2001 Los Indigenas del Paraguay, Ceaduc, Asunción.

Zavala José Manuel

2000 Les Indiens mapuche du Chili : dynamiques inter-ethniques et stratégies de résistance, ${ }^{\prime}{ }^{\prime I I}{ }^{e}$ siècle, L'Harmattan, 